\title{
Research on Simulation Technology of Aircraft Security Operation Turnaround Time
}

\author{
Jianguo Cui ${ }^{1, \mathrm{a}}$, Pengjia Zhou ${ }^{1, \mathrm{~b}}$, Mingyue $\mathrm{Yu}^{1}, \mathrm{Xiaoyu}_{\mathrm{Xu}}{ }^{2}$ and Ruikai Wang ${ }^{2}$ \\ ${ }^{1}$ School of Automation, Shenyang Aerospace University, Shenyang, 110136, China; \\ ${ }^{2}$ Shenyang Aircraft Design \& Research Institute, Shenyang 110035, China; \\ agordon_cjg@163.com, ${ }^{b}$ zhoupengjia0820@163.com
}

\begin{abstract}
Military aircraft again deployed security job preparation time, directly determines whether the aircraft quickly into the operational state, affect the wartime aircraft out of strength, and even decide the outcome of a war. In view of the current analysis of military aircraft again deployed security job preparation time difficult problem, again to military aircraft readiness activities as the research object, in the clear out again the logical relationship of basic security operations, design of military aircraft readiness activities again guarantee process, presents the calculation model of program evaluation and review technique based on readiness time again and its theory, and finite state machine are combined, constructs the computing model of Stateflow turnaround time simulation based on. The clock can be accurately calculated to promote the simulation of military aircraft turnaround time, were tested at last to a certain type of military aircraft real security activities as the example to the model. Results show that created Stateflow military aircraft based on again readiness time calculation model is accurate and reliable, the calculation results and the actual security time error less than 5\%, a better solution to the actual difficult to calculate military aircraft readiness time again the problem of automation level rise provided aircraft again deployed security and decision, which has engineering application value and good prospect.
\end{abstract}

Keywords: Security operation, Turnaround time, PERT, Stateflow, Simulation

\section{Introduction}

Military aircraft again deployed security is to point to that in order to meet the demand of deploying again, take a series of security work in the period when the plane land to release. Mainly includes the flight check, add oil and gas, load mission data and hang arms, munitions, etc., its purpose is to make sure the plane can out in a row. The strength of the plane were very demanded by modern air combat, sortie rates even directly affect the odds, and the intensity of the response is measured by a unit time sortie, this requires a plane again deployed security work must be rapid, efficient, timely and finish accurately. However, due to the out security work again by the battlefield environment, mission requirements, staffing levels, and many other uncertain factors, cause again deployed security operations preparation time calculation difficult[1-3]. Therefore, the research on military aircraft security operations preparation time simulation technology, can clear out again to ensure process, accurate simulation operating out again to ensure preparation time, can provide a reference for the analysis of aircraft support operations preparation time, it has important military significance and research value.

At present, in the field of aircraft integrated engineering research and maintenance support to the development of relatively perfect, but as our army's growing military strength as well as the requirement of combat effectiveness of aircraft is increasing, the demand for aircraft use security research also increasingly urgent. However, due to the use of security content is complex and object different requirements vary, now has not yet 
appeared relatively comprehensive and systematic research. Plane again deployed security as an important content of using security, remains to be further research and improvement on it. Document [4] studied the turnout time again for the aircrafts, which emphasis Stateflow simulation calculation method. Document [5] aviation equipment maintenance preparation time estimation model is established, and the model was solved. Document [6] on the navy airport security process simulation and optimization, and the batch aircraft were simulated, and discusses the different resources set number and the plane out of time.

Based on a certain type of military aircraft is true again deployed security activities as the specific research object, put forward a kind of evaluation technology(Program Evaluation and Review Technique, PERT) based on the plan. Calculation method of the turnout time again, and uses the Stateflow carries on the simulation analysis, finally in this paper, through examples of military aircraft security operations preparation time accuracy and reliability of the simulation analysis method.

\section{Once Again Security Analysis}

\subsection{Security Activities of Logic Analysis}

Logical analysis is based on certain criteria, to make clear the project (activities) between the internal and external relationship process. Aircraft out again preparatory activities logic analysis is simply means through an in-depth analysis of out again all preparatory activities, according to the ground rules and safety regulations maintenance support, make clearly priority of the all preparatory activities, Serial and parallel order process.

Based on ground maintenance inspection and maintenance procedures, in order to make the preparatory time flew again as short as possible, tend to make logic relationship for the Preparatory activities. For aircraft maintenance support out again, specific safeguards activities generally include "charge, fill, plus, hanging, check".

(1) Charge: mainly refers to various types of gas needed to fill the aircraft, such as oxygen, nitrogen, and ordinary high purity nitrogen.

(2) Fill: mainly refers to fill shells, filled shells of different types of aircraft are also different when required to perform different tasks.

(3) Plus: mainly refers to the refueling and affiliated oil, such as hydraulic oil, lubricating oil and so on.

(4) Hung: mainly refers to mount the ammunition, has certain similarities with filled shells, but for different types of aircraft and the existence of certain difference. Fill is mainly for guns weapons filled bullets on the plane, and the hanging is the aircraft mounted missile pod, etc.

(5) Inspection: mainly refers to the crew on the plane were out again make some simply maintenance. The most vulnerable to problems in the site, such as visual inspection, the inlet check, check engine blades, power checks and other activities.

Based aviation maintenance support procedures required: preparatory activities out again, regardless of the order between nitrogen, oxygenation and refueling operations, but not be performed simultaneously; other inspections may be carried out in parallel with the above work; Hanging spring and proofreading of operational requirements and must be performed after the completion of all inspection work; after all work is completed to prohibit re-enter the cockpit, the aircraft would be checked by the pilot.

\subsection{Process Design of Once Again Deployed Security}

After make clearly about the logic relationship of the preparatory activities again, need to design security work flow. Process refers to the arrangement order of 
preparatory activities again. The basic process of the plane out again deploy security are: after security task command is received, crew and support equipment, devices, equipment, etc. in place quickly, and the plane out again towing the security line. Then, it is carried out by refueling, inflatable oxygenation, mounted weapons and the maintenance and inspection activities. After the completion of security work, carried out by a mechanic working on the review, and wait for the pilot check and acceptance, the plane ready to take off after the result is good, then the process of out again is over[11-13]. In this paper, according to aviation maintenance procedures designed to protect military aircraft out again assurance processes shown in Figure 1.

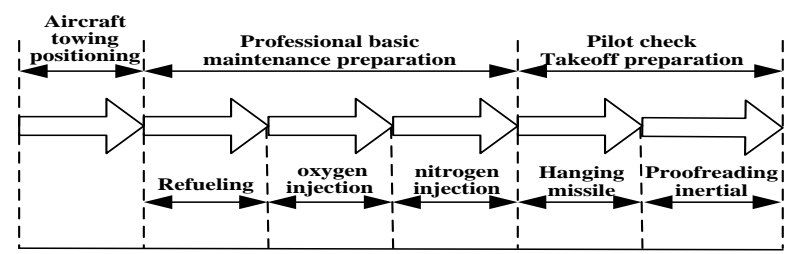

Figure 1. Support Process of Military Aircraft Turnaround

\section{Calculation of Preparatory Time Out Again}

\subsection{Construction of PERT Network Model}

PERT is the technology to plan and evaluated that use the method of network analysis. Its main feature is a means to deal with the uncertainty of the duration of the procedure [7]. PERT network depict the order and time of the activities included in the project by arrows through the relationship between activities to build the network model and gives the analysis and calculation. As support process of aircraft turnaround have a heavy work, and the duration of the job to meet certain probability distribution, very suitable for the security process are described in PERT, and it has the advantages of simple and intuitive [8-9]. PERT build out preparatory time out again computing model as follows:

Step 1: Make sure protect the preparatory work required for the security job is completed, and according to security procedures and process design results, clear logical relationship of all activities, the most optimistic time, required to collect and collect the activities of the most pessimistic time and the most likely time parameter data. According to the $\beta$ distribution rule, using the three estimation formula $(t=a+4 c+b / 6, \mathrm{t}$ as the duration, a as the most optimistic time, $\mathrm{b}$ as the most pessimistic time, $\mathrm{c}$ as the most likely time) calculated the duration of the activities and listed the preparatory time out again.

Step 2: According to the drawing rules of the network planning chart, and circles represent events, an arrow represents the active job, proper use the virtual job describe corresponding logic relation of activities, from the beginning to the end get the network diagram of support process of military aircraft turnaround, known as the PERT network model.

Step 3: To estimate the time parameters of the network graph of each job, and use a certain method of calculation to obtain the total estimated time to complete the support activities. And the time parameters of the network diagram includes the job of the earliest and latest time, the activities of the earliest start and end times, latest start and end time, activities and items total time difference and so on. Assuming $T_{E(j)}$ as the earliest time of job indicated by network diagram arrow, $T_{L(j)}$ as the latest time arrow matters, $T_{E(i)}$ as the earliest time of nock work, $T_{L(i)}$ as the latest time nock 
matters, $t_{e(i j)}$ as the time of support activities, the network graph parameters calculated as follows:

(1) The earliest time of job. It refers to from the starting time to the time that the longest line of the job, the job can not be started before this time, expressed as $T_{E(j)}=\max \left[T_{E(j)}+t_{e(i, j)}\right]$.

(2) The latest time of job. It refers that not affect the start time of each job. The latest time of terminal job is equal to the total time of security, expressed as $T_{L(j)}=\min \left[T_{L(j)}-t_{e(i, j)}\right]$.

(3) The earliest start time of support activities. Only refer to activities must begin at the end of predecessor activities, also called active earliest start time or the earliest starting period, expressed as $T_{E S(i j)}=T_{E(i)}$.

(4) The earliest finish time of support activities. Refers to the earliest start time of the activities of and sum with the duration, expressed as $T_{E F(i j)}=T_{E S(i j)}+t_{e(i j)}$.

(5) The latest start time of support activities. Refers to the latest time must begin that activities having multiple tight before working under the conditions do not affect the start of the period, expressed as $T_{L S(i j)}=T_{L F(i j)}-t_{e(i j)}$.

(6) The latest finish time of support activities. Refers to the latest starting time of the activities sum with the duration of activity, expressed as $T_{L F(j)}=T_{L(j)}$.

(7) Total float of activities. Refers to without affecting the total completive time of the support activities and the earliest start time of tight before, the activities maybe delay the time of maximum, The larger the difference would indicate the time of the activity will maneuver more, expressed as $R(i j)=T_{L S(i j)}-T_{E S(i j)}$ or $R(i j)=T_{L F(i j)}-T_{E F(i j)}$.

(8) The time difference of job. Refers to the difference between the earliest time and the latest start time of the matter, represents the maximum time of maneuvering, the job time difference is zero are called key jobs, expressed as $R_{i}=T_{L(i)}-T_{E(i)}$.

Step 4: Find out the jobs time difference are zero in the network, and add their duration, we will get the total preparatory time of military aircraft turnaround.

\subsection{PERT Simulation based on Stateflow}

As manual calculation of the PERT network diagram is complicate, and the results of each calculation can only get once, unable to meet the needs of statistical analysis, so it will be converted to Stateflow simulation model to improve the speed and accuracy of calculation. Stateflow is a kind of design simulation tool that is graphical and integrated in Simulink simulation system in MATLAB, using the principle of finite state machine to achieve, with logic is simple and clear, the discrete event analysis ability and many other advantages [10]. To the calculation of simulation for the preparatory time of military aircraft turnaround, based on the PERT model that established, to convert it to Stateflow simulation model, the work, time, path and logic relation convert to entity in Stateflow, data, events and actions, using clock gradually run the simulation model automatic, can effectively overcome the logic relation under complex circumstances and the difficult problem of the calculation of PERT network. The procedure that calculation of PERT model using Stateflow simulation is as follows:

Step 1: Determine the entity. Stateflow is an object-oriented simulation software, so first is determine the system entity. The system entity is all the assignments the support activities of aircraft turnaround, the jobs will be shown in block diagram, In addition, statistical frame and graphics functions are needed to build the simulation box, for achieve the record of the simulation data and compute the duration of jobs. 
Step 2: Establish event. Stateflow simulation model run by an event to promote, so it need to establish an external event timer input as the system clock. Meantime each entity will need some start event and end event to promote its run, the number of start events should equal to the number of jobs immediately before the process of, the number of end events should equal to the number of steps after the process. Start events can be named start, start 1 and so on, end events can be named END, ENDl and so on. However, no first job immediately preceding process, simply add the end of the event, there is no tight after the last step of a job, just add start event. Also, need to add an internal event LE and EL, each job is triggered between the Early and Late state transitions, which is convert the order and reverse of jobs.

Step 3: Adding data. Stateflow operands are data entity. The input data for the duration of the protection of jobs, it is calculated by the system of distribution on the basis of performance function. The output data is sent to protect the total time again. In addition, the need to add an internal data for each job, the internal data is the most optimistic safeguard jobs, the most pessimistic, most likely time and other parameters.

Step 4: Simulation. After completing the Stateflow block diagram structures, after add data, need to set up the simulation system in Simulink, add a trigger pulse, oscilloscopes, memory and other components needed, you can achieve aircraft run again and preparation time's calculate output.

\section{Experimental Study}

In Table 1, it is the support activities about a military aircraft out again.

Table 1. The Support Jobs of a Military Aircraft Turnaround

\begin{tabular}{|c|c|c|c|c|c|c|}
\hline Process & Job name & Preceding activity & $\begin{array}{l}\text { Optimistic time } \\
\text { (minutes) }\end{array}$ & $\begin{array}{l}\text { Pessimistic time } \\
\quad \text { (minutes) }\end{array}$ & $\begin{array}{l}\text { Possible time } \\
\text { (minutes) }\end{array}$ & Professional \\
\hline $\mathrm{A} 1$ & Traction & - & 2 & 4 & 3 & - \\
\hline A2 & Tethered aircraft & A1 & 1 & 3 & 2 & All the staff \\
\hline A3 & Dismantling missiles & $\mathrm{A} 2$ & 3 & 5 & 4 & Ordnance \\
\hline A4 & Data download & A2 & 2 & 4 & 3 & ad hoc \\
\hline A5 & Machine visual inspection & A3 & 3 & 4 & 4 & Mechanical \\
\hline A6 & $\begin{array}{c}\text { Avionics appearance and } \\
\text { power check }\end{array}$ & A5 & 5 & 6 & 6 & Avionics \\
\hline A7 & $\begin{array}{c}\text { Ordnance appearance and } \\
\text { power check }\end{array}$ & A5 & 6 & 7 & 6 & Ordnance \\
\hline $\mathrm{A} 8$ & $\begin{array}{c}\text { Ad hoc appearance and } \\
\text { power check }\end{array}$ & A5 & 5 & 7 & 6 & ad hoc \\
\hline A9 & Inlet check & A6、A7、A8 & 3 & 4 & 4 & Mechanical \\
\hline A10 & Install the drogue & A3 & 3 & 5 & 4 & Mechanical \\
\hline A11 & Extra auxiliary fuel tanks & A3 & 2 & 5 & 4 & Mechanical \\
\hline $\mathrm{A} 12$ & fuel filling & A9, A11 & 5 & 6 & 6 & Mechanical \\
\hline A13 & Oxygen injection & A12 & 3 & 4 & 3 & ad hoc \\
\hline A14 & Nitrogen injection & A13 & 2 & 4 & 3 & Mechanical \\
\hline A15 & Hanging missile & A10、A14 & 5 & 7 & 6 & Ordnance \\
\hline A16 & Proofreading inertial & A15 & 4 & 7 & 5 & Avionics \\
\hline A17 & Load task data & A3、A4 & 3 & 5 & 4 & ad hoc \\
\hline A18 & Pilot check & A16、A17 & 2 & 3 & 2 & - \\
\hline A19 & Flight inspection & A18 & 2 & 3 & 2 & - \\
\hline
\end{tabular}




\begin{tabular}{lllllll}
\hline A20 & Take off & A19 & 2 & 4 & All the staff \\
\hline
\end{tabular}

\subsection{PERT Model Constructed Out Again}

According to the operation out again and logical relationship shown in Table 1, constructed PERT model shown in Figure 2.

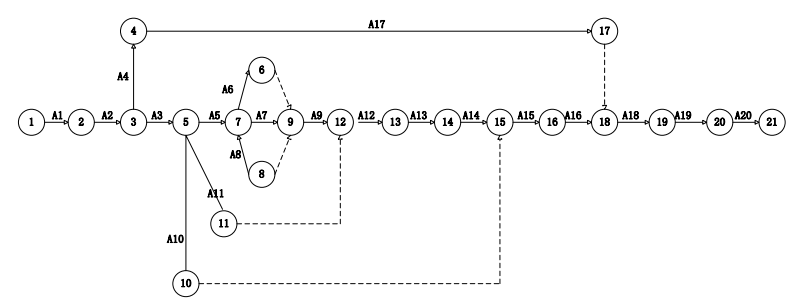

Figure 2. The PERT Model of Military Aircraft Turnaround

\subsection{Calculation based on Stateflow of the Preparation Time Out Again}

Simulation based on MATLAB Stateflow modeling steps; first determine the simulation system entities. In this case, the aircraft out again protect the activities of the entity is protect jobs, according to the Table 1 for a description of the job, build planes out again Stateflow simulation diagram shown in Figure 3, including parallel block diagram A1, A2, A3, .., A20, A, ST and ED.

Wherein, A is the statistics block, it is used to control a clock and recording data of simulation, A1 A20 is uses to describe the state of 20 prepared jobs. ST and ED control switching step of positive and reverse state. The role of function $y=C(a, b)$ is adopts three-time estimation method to generate jobs in line with the duration of $\beta$ distribution, shown in Figure 4.

In each job status diagram, describe the sequence of steps to establish the state of early occurrence, established state Late description reverse process occurs. At the same time, need to establish state of on and state of off for state Early and Late to describe the work of active and inactive states. Meantime, need to establish a virtual state of Tm aim at various jobs, describe the process immediately after it before or after all the work is completed immediately, before it enters the state trigger.

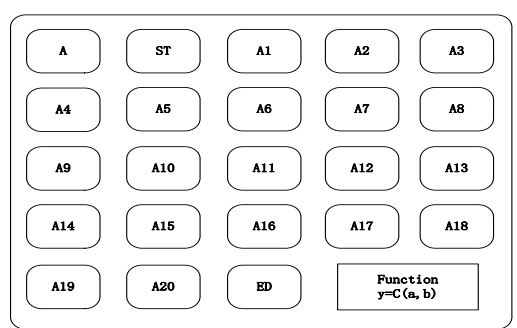

Figure 3. The Stateflow Block Diagram of Aircraft Turnaround

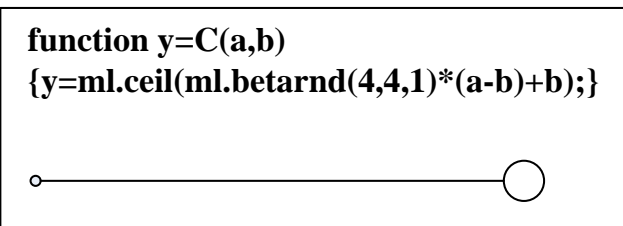

Figure 4. Function Block Diagram of $y=C(a, b)$ 
This paper based on the operation readiness activities--inlet check again (A9) work process and state of the operation situation in reference to the description of the whole system.

A9's stateflow diagram shows in Figure 5. When its status is triggered, A9 first to be triggered to its Early box of default off state, while receiving their precedence A6, A7, A8 send a trigger signal. Tm status is virtual buffer status of the ahead three immediately preceding process established, until all the trigger signal has been received, A9 has been entered into on. $s$ is earliest start time variables, which recording the instantaneous time when entered into the state. Then, through the function of $y=C(4,3)$, gets the time $s$ of the job duration of A9. The simulation of the running program will trigger the logic function of after $(t$, timen $)$, so A9 went back to the off state. Variable $e$ records the moment of exit about on state, that is the time of A9 process early ends. At the same time, activated the start event and send it to A12 process immediately steps after. When all processes run once after the positive sequence, in ED control box trigger event EL to promote reverse run, at this point A9 directly into Late box off default state and wait for it to close after step A12 END send trigger signal, after received signal END signal, turn to on state, by $m$ variables to record the moment as the latest start time A9 process. Similarly, when the trigger function of after $(t$, timen $)$, A9 back off state and use variable $n$ recording the time of A9's latest finish time, and activate the END event of its immediately preceding step A6, A7, A8, By this time, complete the simulation of reverse. The rest of the process is similar to the process of running and A9; this is no longer introduced one by one.

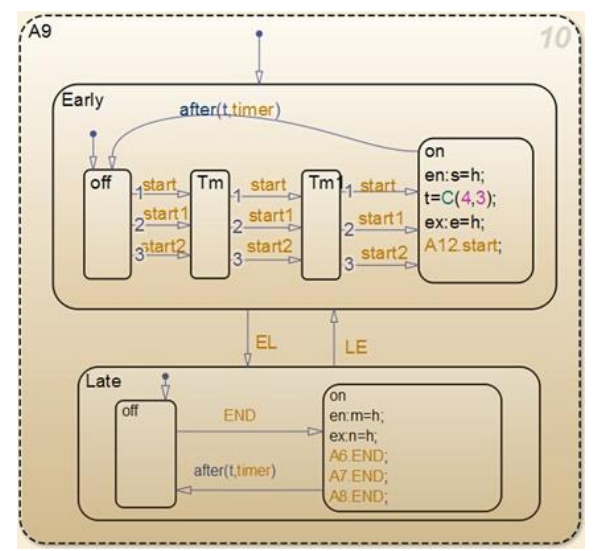

Figure 5. The Status Block Diagram of A9 Job

Taking the ED control box as an example, illustrate the positive and reverse conversion run, as shown in Figure 6.

After the start of the simulation, ED enter default off state, when the positive sequence runs to the job A20, start trigger ED status box, ED into the on state, while triggering internal events EL, so the job A1 A20 into the Late default off state, and then ED return to the off state, sent a trigger event to the A20, start running in reverse. The control box ST is similar to ED, control the simulation switching from reverse to positive, here we are not narrative.

The operation of the entire system is based on Simulink simulation environment, therefore, Stateflow state block diagram needed to collocate the corresponding auxiliary modules form a complete simulation model. Figure 7, the auxiliary module has data storage module (simout), responsible for each simulation result data $\mathrm{T}$ stored in the workspace of the matrix simout; the pulse trigger (PulseGenerator), 
mainly used to trigger the timer event; data display module (Display), used to display data of simulated results.

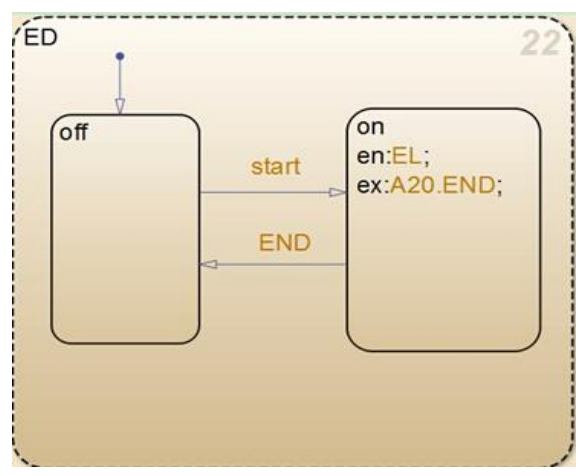

Figure 6. The ED Control Block Diagram

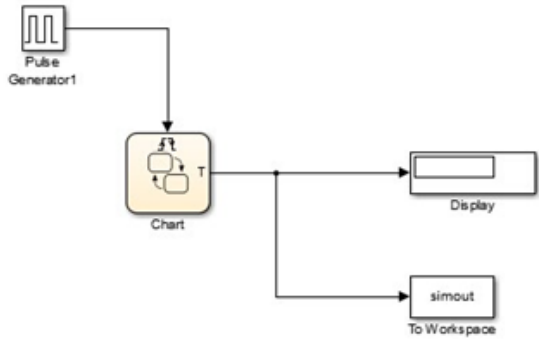

Figure 7. The Simulink Simulation System

Set the simulated system to run 200 times, statistics and analyzed the results, the results shown in Figure 8.

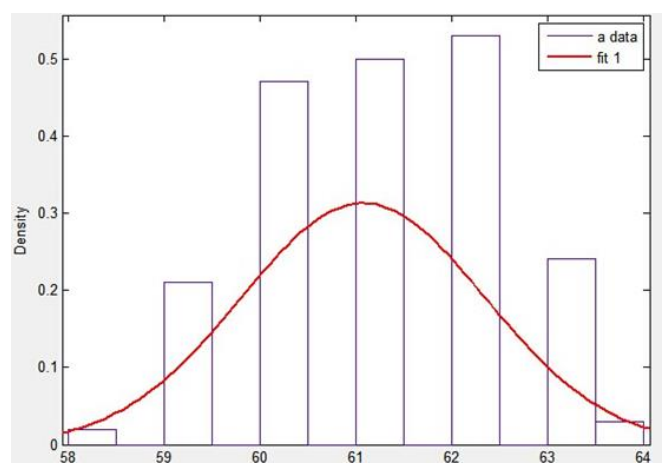

Figure 8. Simulation Results Distribution

Gain the aircraft out again preparation time expectation and variance are 61.075 minutes and 1.62751 by calculated. Expectation is the average simulation time, referring to the aircraft floating out again time to prepare for the mid-point range to about 61.075 changes. The variance is the degree of deviation between the simulation results and the expected value, variance, the more dispersed data, the results more imprecise, otherwise, more accurate and vice versa. Compared with real time protection and security department, out again ready time error less than $5 \%$, meet the requirements of preparation time calculation error. 


\section{. Conclusion}

Analyzing logical relations of military aircraft out again security active, confirm logical relationship among the various safe guard jobs, and on this basis, the process is designed, provide a theoretical basis for the analysis for the military aircraft out again protected active. Preparation time out again created a comprehensive computational model contains a basis for protection on the job logic, considering the volatility of the protection operation time, making simulation results more fit reality, simulation model constructed scientific, rational, so that the calculated results more accurate. Preparation time out again created a comprehensive computational model contains a basis for protection on the job logic, considering the volatility of the protection operation time, making simulation results more fit reality, simulation model constructed scientific, rational, so that the calculated results more accurate.

The results show that the simulation analysis method of military aircraft protected jobs preparation time can effectively solve the problem of preparation time flew again difficult to calculate, and consistent with the simulation results with the actual situation, can provide guidance for aircraft deployed to protect again and decisionmaking, can further enhance the aircraft again reliability and automation level of protection and making out. This method can also be applied to other weapons' protected jobs preparation time analysis, has certain significance.

\section{Acknowledgments}

This work is supported by the Aeronautic Science Foundation of China (ID2010ZD54012), the Defense Industrial Technology Development Program of China (IDA0520110023) and the Defense Technology Advance Research Foundation of China (IDZ052012B002), the Liaoning Province Natural Science Foundation of China(ID2014024003).

\section{References}

[1] M. Spirtas, "What it takes: Air Force Command of Joint Operations", Project Air Force, RAND, (2009).

[2] C. M. He, F. K. Kong and S. Y. Ren, "Discussion of Problems about Weaponry Operational Support", Journal of Armored Force Engineering Institute, vol. 3, no. 15, (2001), pp. 12-16.

[3] Y. Wei, R. Kang and H. L. Cheng, "Calculation method for military aircraft's turnaround time", Journal of Beijing University of Aeronautics and Astronautics, vol. 12, no. 34, (2008), pp. 1214-1217.

[4] F. Ren, X. Z. Lv and Y. L. Yu, "Simulation and calculation method of military aircraft's turnaround time", Project Management Technology, vol. 3, no. 8, (2010), pp. 28-31.

[5] Y. Wang, F. Liu and X. D. Xu, "Research on Estimating Method of Flight Line Maintenance Time for Aviation Equipment", Mathematics in practice and theory, vol. 8, no. 42, (2012), pp. 185-189.

[6] S. H. Wang, "Simulation and Optimization for Flight Maintenance Process of Navy Airport", Harbin: Engineering University, School of Mechanical Engineering, (2012).

[7] G. C. Zhao, "The Research on Multi-resource Constrained Project Scheduling Problem", Qinhuangdao: Yanshan University, School of Economics and Management, (2011).

[8] J. M. H. Velasco, R. H. Pleguezuelo and J. R. Dorp, "Revisiting the PERT mean and variance", European Jounal of Operational Research, vol. 210, (2011), pp. 448-451.

[9] S. Baradaran, S. M. T. Fatemi Ghomi and M. Mobini, "A hybrid scatter search approach for resourceconstrained project scheduling problem in PERT-type networks", Advances in Engineering Software, vol. 41, (2010), pp. 966-975.

[10] F. Rev, Y. L. Yu and X. Z. Lv, "Research on equipment maintenance process simulation based on Stateflow", Computer Engineering and Applications, vol. 21, no. 47, (2011), pp. 217-219.

[11] J. C. Zhao, H. Y. Zhu and W. X. Wang, "Operations Research on Technical Support for Aviation Material”, Beijing: National Defense Industry Press, (2011), pp. 126-149.

[12] J. L. Li and Y. G. Tao, "Optimization of Designing Aircraft Flying Mission Support System”, Industrial Engineering, vol. 3, no. 12, (2009), pp. 85-88.

[13] Y. P. Wang, "Field Maintenance Scheduling and Operation Optimization Simulation", Harbin: Engineering University, School of Mechanical Engineering, (2012). 
[14] X. F. Luan and J. Xie, "Research on Multi-aircrafts Preparation Process Based on Simulation Optimization", Computer and digital engineering, vol. 12, no. 38, (2010), pp. 50-53.

\section{Authors}

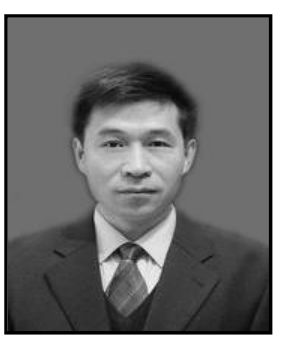

Jianguo Cui, he was born in August, 1963. He is from Liaoning, China. $\mathrm{He}$ is a professor and $\mathrm{PhD}$ supervisor in Shenyang Aerospace University. His main research interest is aircraft health diagnosis, prediction and comprehensive health management.

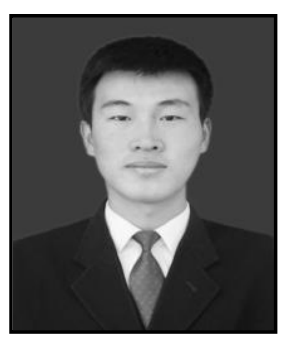

Pengjia Zhou, he was born in August, 1990. He is a graduate student in Shenyang Aerospace University. His main research interest is aircraft comprehensive health management and security decision. 